\title{
Diagnostic value of serum precipitins to mould antigens in active hypersensitivity pneumonitis
}

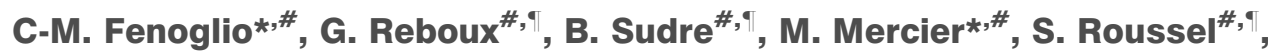 \\ J-F. Cordier ${ }^{+}$, R. Piarroux ${ }^{\#, \uparrow}$ and J-C. Dalphin ${ }^{\#, \S}$
}

ABSTRACT: Serum precipitins have a controversial diagnostic value in hypersensitivity pneumonitis (HP). The present authors' objective was to assess their diagnostic value by developing scores from a panel of specific antigens tested by two techniques (electrosyneresis and double diffusion) to discriminate active HP from other interstitial lung diseases.

Consecutive patients presenting with a condition for which HP was considered in the differential diagnosis were included in the study. All patients underwent the same standardised diagnostic procedure, including precipitin tests performed in routine conditions. Clinical manifestations, bronchoalveolar lavage and high-resolution computed tomography defined the presence or absence of HP. Receiver-operating characteristic curves and logistic regression were used to develop the serological scores.

A total of 122 patients (including 31 cases of HP) were included in the study. Five antigens from the panel were selected for the serological scores (Absidia corymbifera, Eurotium amstelodami, Wallemia sebi, Saccharopolyspora rectivirgula and mesophilic Streptomyces sp.). Electrosyneresis was more discriminative than the double-diffusion technique. Predictive negative values varied $81-88 \%$ and predictive positive values varied $71-75 \%$ for prevalence of HP $20-35 \%$.

In conclusion, serological scores using a panel of relevant antigens may guide both biological and clinical practice in areas of high prevalence of hypersensitivity pneumonitis.

KEYWORDS: Diagnosis, hypersensitivity pneumonitis, precipitins, prospective study, serology

\footnotetext{
西
} nterstitial lung diseases (ILDs) represent a large group of diseases that includes hypersensitivity pneumonitis (HP). HP, also called extrinsic allergic alveolitis, is a syndrome that results from an immunologically induced inflammation of the lung parenchyma in response to inhalation of a large variety of antigens to which subjects have been previously sensitised. Despite its apparently low prevalence, the impact of HP in individuals of all ages throughout the world continues to be a major concern [1]. The most common forms are farmers' lung (FL) and bird breeders'/fanciers' lung (BBL). Their prevalence rates and distribution vary widely among countries and geographical locations due to local customs and occupational and climatic conditions [2]. Differential diagnosis between HP and other ILDs is difficult and relies on an array of signs or criteria [1,3-5]. Although nonconsensual, these diagnostic indicators generally include the following: 1) clinical symptoms and signs developed in an appropriate environment; 2) the presence of precipitating antibodies against offending antigens;
3) consistent chest radiograph or high-resolution computed tomography (HRCT); 4) bronchoalveolar lavage (BAL) lymphocytosis; 5) decreased carbon monoxide diffusion capacity; and/or 6) a granulomatous reaction on lung biopsies; and/or 7) a positive inhalation challenge. Among the above-mentioned indicators, the diagnostic value of serum precipitins has long been controversial [6], particularly because of their lack of sensitivity and, especially, of specificity [7]. These limitations may be due to the use of poorly purified antigens, the lack of the specific inciting antigen in the test panel, inappropriate techniques [8] or, in epidemiological studies, the use of inappropriate control groups [5, $9,10]$. Despite the pitfalls presented above, a recent multicentre study by LACASSE et al. [4] identified serum precipitins as a significant predictor of $\mathrm{HP}$, regardless of exposure (odds ratio 5.3 (95\% confidence interval (CI) 2.7-10.4)). However, the choice of antigens tested and the use and interpretation of tests were left to each investigating centre according to their usual local practice; thus, the diagnostic value of precipitins could not be
AFFLLIATIONS

*Dept of Biostatistics, University of Franche-Comte, and ${ }^{\#}$ SERF (Santé et Environnement Rural, University of Franche-Comté) Group

"Dept of Mycology and

${ }^{\S}$ Dept of Respiratory Diseases, CHU Besançon, Besançon 'GERM'O'P, Dept of Respiratory Diseases, Hôpital Louis Pradel, Lyon, France.

CORRESPONDENCE

G. Reboux

Dept of Mycology

Jean Minjoz University Hospital

Boulevard Fleming

25030 Besançon Cedex

France

Fax: 33381668914

E-mail: Gabriel.reboux@ufc-

chu.univ-fcomte.fr

Received:

January 042006

Accepted after revision:

December 122006

STATEMENT OF INTEREST

None declared.

European Respiratory Journal Print ISSN 0903-1936

Online ISSN 1399-3003 
assessed reliably. With the objective of developing serological scores to discriminate active HP from other ILDs, the present authors conducted a prospective cohort study of patients presenting in their department with a suspected diagnosis of HP. Subjects were tested by two serological techniques (electrosyneresis and double diffusion) for a panel of seven antigens. The choice of both immunological methods and the antigenic panel was based on previous studies aimed at identifying the best immunological set for diagnosing HP in the present study's region $[11,12]$.

\section{METHODS}

\section{Patients}

From January 1, 1999, consecutive patients were recruited aged $\geqslant 18$ yrs presenting in the Dept of Respiratory Diseases (University Hospital of Besançon, Besançon, France) with a pulmonary syndrome for which HP was considered in a differential diagnosis (patients diagnosed in 1999 were also included in the study by LACASSE et al. [4]). The proportion of $\mathrm{HP}$ and other ILDs was unspecified $a$ priori.

All patients underwent the same standardised procedure (discussed later). Only HP cases presumably due to moulds (fungi and thermophilic actinomycetes) were included, since BBL, chemical HP and hot-tub lung are due to antigens of a nature different from those of other HP caused by microorganisms.

\section{Diagnostic criteria}

Since patients were to be classified as either HP or non-HP (other ILD), and in the absence of a recognised gold standard, the following diagnostic criteria were used for $\mathrm{HP}$ according to those of a previous HP study [4]. 1) Compatible clinical manifestations; 2) BAL lymphocytosis ( $\geqslant 30 \%$ for non- and exsmokers and $\geqslant 20 \%$ for current smokers); and 3) bilateral ground glass or poorly defined centrilobular nodular opacities. When the association of BAL and HRCT did not yield a reliable diagnosis of $\mathrm{HP}$, the presence of a positive specific provocation test [13], an improvement after avoidance of supposed antigens, or suggestive features on transbronchial biopsies was required. Patients underwent surgical lung biopsy when the previously described diagnostic procedure failed to yield a diagnosis. Classical histopathological descriptions were used [14]. Patients considered to have residual HP (a chronic and inactive form in which BAL lymphocytosis has disappeared) were classified in the non-HP group. When HP was diagnosed, the patient's exposure to antigenic sources of HP was then specifically considered in order to determine the nature of $\mathrm{HP}$, e.g. FL, other mould-induced occupational $\mathrm{HP}$, home $\mathrm{HP}$ caused by moulds, etc.

When a complete diagnostic procedure set did not lead to a reliable classification (for example, difficulties in interpreting HRCT), cases were reassessed by an independent ILD expert (J-F. Cordier), who considered, in particular, BAL and HRCT data but also data on clinical history, physical examination, spirometry and diffusing capacity of the lung for carbon monoxide (or the results of a 6-min walk test) to reach a diagnosis. If diagnosis remained inconclusive, patients were excluded.

\section{Precipitins tests}

The choice of antigenic panel was based on the recognised causes of FL and HP due to moulds at an international [15, 16] and local level [11]. The antigenic panel tested included Absidia corymbifera, Eurotium amstelodami, Wallemia sebi, Aspergillus fumigatus, Saccharopolyspora rectivirgula, mesophilic Streptomyces sp. and hay extract. Antigen extracts were produced and tested as described in a previous article [11]. The immunological methods used were agar gel double diffusion and electrosyneresis on cellulose acetate, which, as shown by a recent study, are more relevant than ELISA and immunoblots in discriminating FL from healthy exposed dairy farmers in the present study's region [12]. Briefly, electrosyneresis was carried out as follows. Cellulose acetate sheets (Sartorius, Göttingen, Germany) were placed in the electrophoresis vat filled with buffered Tris glycin solution at $\mathrm{pH} 8.8$; $15 \mu \mathrm{L}$ of each serum was placed on three spots on the anode side and a $15-\mu \mathrm{L}$ line of antigen was placed on the cathode side. A current $(110 \mathrm{~V})$ was then applied for $75 \mathrm{~min}$ and the sheets were stained with Coomassie blue. Serological tests were performed and interpreted (by counting the number of arcs) under routine conditions by four different experienced technicians and a supervisor blinded to the patients' diagnoses and exposure.

\section{Statistical analysis}

Characteristics of HP and non-HP patients were compared using the two-tailed Fisher's exact test for dichotomous variables and the unpaired t-test for continuous variables.

The serological results for each antigen were interpreted as a number (xa) of precipitating arcs (discrete quantitative variable). They were used, either as discrete (xa) or dichotomous variables (xd), to develop serological scores. The selection of antigens for multivariate analysis was based on receiveroperating characteristic (ROC) curves to each antigen [17]. Efficiency was used to determine the positivity threshold of each antigen. Serological scores were then constructed for electrosyneresis and double diffusion using multivariate logistic regression (full model) and stepwise logistic regression (predictive model). Coefficients $(\beta)$ were estimated with their $95 \%$ CIs by multivariate analysis. Scores (Sa and $\mathrm{Sd}$ ) with antigenic results as discrete or as dichotomous variables $(\mathrm{Xd}$ : positive or negative) were expressed respectively, as follows.

$\mathrm{Sa}=\beta_{0}+\sum \beta_{\mathrm{a} X a}$, with $\mathrm{X}_{\mathrm{a}}=$ number of precipitating arcs for each antigen tested

$S d=\beta_{0}+\sum \beta d X d$, with $X_{d}=0$ (negative) or 1 (positive) for each antigen tested

The stability of the models was studied using the bootstrap method [18]. In total, 1,000 random bootstrapped samples (size=study sample size) were generated, and a robust calculation of $95 \%$ CIs and coefficient of variation $(\mathrm{CV})$ of estimated parameters $(\beta)$ was obtained.

ROC curves were constructed and areas under the curves (AUC) were compared for electrosyneresis and double diffusion in order to determine the best immunological method.

Thresholds of serological score positivity for different HP prevalence were determined on ROC curves [17], and their associated predictive values were calculated. The serological 
score results, expressed as either positive or negative, were then studied in association with patients' exposure and smoking status.

\section{Estimated accuracy of serological scores}

With the hypothesis of sensitivity (Se) and specificity (Sp) values between $70-90 \%$ and an HP expected prevalence of $35 \%$ (in the present authors' centre), 120 patients needed to be included in the study in order to obtain an accuracy of $\sim 10 \%$ for Se and Sp.

\section{RESULTS}

\section{Patients}

Between January 1999 and April 2005, 162 patients were enrolled in the present study. Of these, 40 were excluded for the following reasons: BBL $(n=3)$, hot-tub lung $(n=2)$; unavailable or uninterpretable BAL $(n=3)$; serological results unavailable $(n=30)$ and indeterminate diagnosis $(n=2)$. The independent investigator reassessed eight cases, three of which were classified in the HP group and four in the non-HP group; one was inconclusive. Thus, 122 patients were included, comprising $31 \mathrm{HP}$ (22 FL, three HP due to nonprofessional farming activities (sheep and horses), two home HP due to moulds, one HP due to nonprofessional sawing (mould from sawdust), one cheese-workers' lung, and two HP with no identified exposure) and 91 non-HP ILDs (41 idiopathic interstitial pneumonia (33 idiopathic pulmonary fibrosis, three cryptogenic organising pneumonia, two acute interstitial pneumonia, two respiratory bronchiolitis ILD, one desquamative interstitial pneumonia), 18 sarcoidosis, seven druginduced interstitial pneumonia, four pneumoconiosis, four eosinophilic interstitial pneumonia, three collagen vascular disease, three left heart failure, two histiocytosis, two neoplasic interstitial pneumonia, two infectious interstitial pneumonia, two residual form of HP and three undetermined ILD).

\begin{tabular}{lccc} 
TABLE 1 & Description of patients' characteristics & \\
& HP \% & Non-HP \% & p-value \\
\hline Patients n & 31 & 91 & \\
Age yrs & $52.7 \pm 14.3$ & $62.4 \pm 15.4$ & $0.003^{\#}$ \\
Sex & & & \\
$\quad$ Male & $19 \pm 61.3$ & $54 \pm 59.3$ & $1.0^{\circ}$ \\
$\quad$ Female & $12 \pm 38.7$ & $37 \pm 40.7$ & \\
Exposure to mould and & & & \\
$\quad$ actinomycetes & & & \\
$\quad$ Yes & $29 \pm 93.5$ & $19 \pm 21.1$ & $<0.0001^{*}$ \\
$\quad$ No & $2 \pm 6.5$ & $71 \pm 78.9$ & \\
Tobacco exposure & & & \\
$\quad$ Nonsmoker & $27 \pm 87.1$ & $47 \pm 52.8$ & $0.0006^{*}$ \\
$\quad$ Smoker & $4 \pm 12.9$ & $42 \pm 47.2$ & \\
$\quad$ Current smoker & $0 \pm 0.0$ & $9 \pm 10.1$ & \\
$\quad$ Ex-smoker & $4 \pm 12.9$ & $33 \pm 37.1$ & \\
\hline
\end{tabular}

Data are presented as mean $\pm \mathrm{SD}$, unless otherwise stated. HP: hypersensitivity

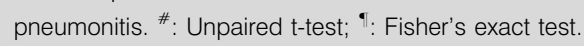

Patients from the HP group were significantly younger, less frequently smokers and more frequently exposed than the patients from the non-HP group (table 1).

\section{Selection of candidate antigens for serological scores}

ROC curves for A. fumigatus and hay extract were respectively nondiscriminative (AUC values 0.54-0.55) and uninterpretable. Therefore, threshold values for these antigens were not determined and they were not used in multivariate analysis to develop serological scores. Positivity thresholds for each antigen for the two immunological methods are indicated in table 2.

\section{Development of serological scores}

ROC curves associated with electrosyneresis scores were more discriminative than those associated with double diffusion, regardless of the type of variables (fig. 1). Similarly, full models appeared to be more relevant than predictive models. Only results concerning electrosyneresis with the full model are presented. Estimated coefficients used to calculate serological scores and their CIs are indicated in table 3. Coefficients of variation from bootstrap re-sampling showed a moderate stability of estimated parameters (table 3 ).

\section{Performances of serological scores}

The positivity thresholds of serological scores according to different prevalence rates $(20,25$ and $35 \%)$ of $\mathrm{HP}$ and their diagnostic values for electrosyneresis are presented in table 4 . Performances of scores with discrete variables were equivalent to those with dichotomous variables for negative predictive

TABLE 2 lut-off values of serological results (number of
precipitating arcs) by antigen and immunological
techniques
Antigens

Double diffusion

Electrosyneresis

$\begin{array}{lc}\text { Absidia corymbifera } & 1 \\ \text { Eurotium amstelodami } & 1 \\ \text { Wallemia sebi } & 1 \\ \text { Aspergillus fumigatus } & \mathrm{ND} \\ \text { Saccharopolyspora } & 1 \\ \quad \text { rectivirgula } & \\ \text { Streptomyces mesophilic } & 1 \\ \text { Mouldy hay extract } & \mathrm{ND}\end{array}$

$\begin{array}{lc}\text { A. corymbifera } & 1 \\ \text { E. amstelodami } & 2 \\ \text { W. sebi } & 2 \\ \text { A. fumigatus } & \mathrm{ND} \\ \text { S. rectivirgula } & 1 \\ \text { Streptomyces mesophilic } & 2 \\ \text { Mouldy hay extract } & \mathrm{ND}\end{array}$

ND: nondiscriminative antigen. To transform serological results from number of arcs (discrete variable) to positive or negative (dichotomous variable) using this table, serological results must be coded as 0 (negative) if the number of arcs is below the cut-off value, and as 1 (positive) if the number of arcs is equal to or above the cut-off value. 

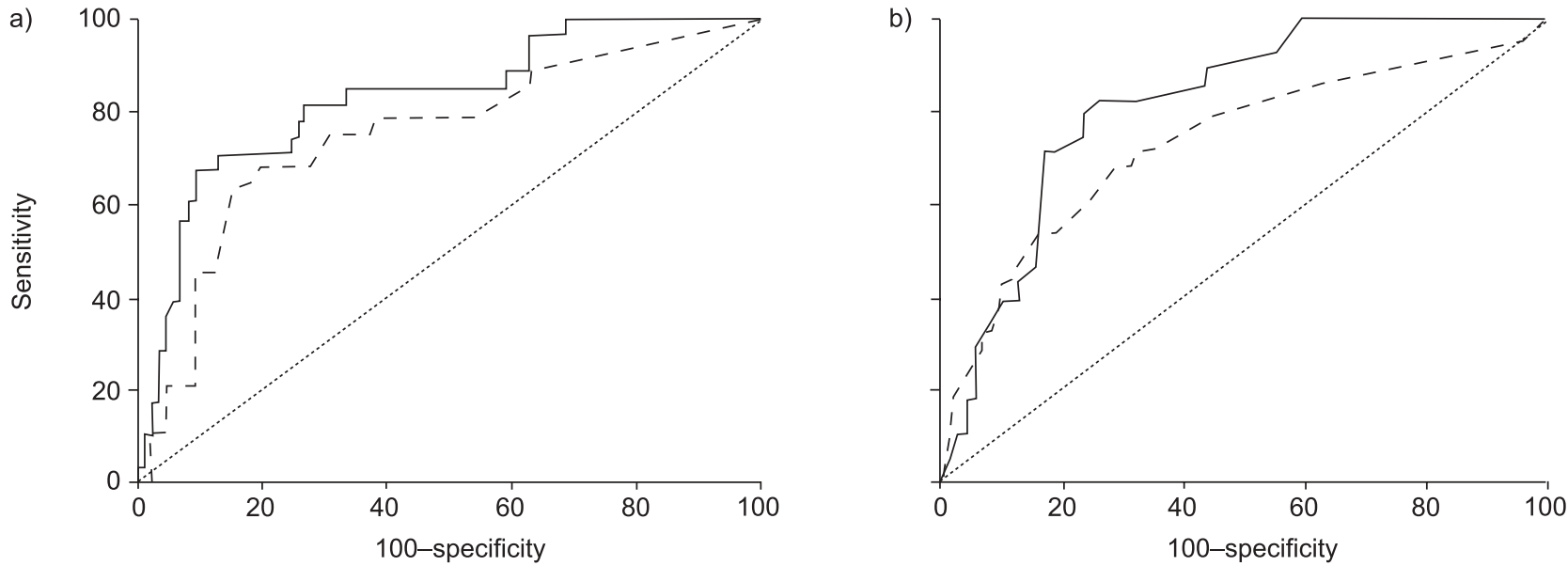

FIGURE 1. Receiver-operating characteristic (ROC) curve for electrosyneresis and double-diffusion showing a) discrete variables, full model ( $p=0.247$; area under the curve (AUC) with electrosyneresis $(A e)=0.830 ; A \cup C$ with double diffusion $(A d)=0.751)$ and $b)$ dichotomous variables, full model $(p=0.260 ; A e=0.814 ; A d=0.730)$. The $A \cup C$ is equivalent to the probability that a patient drawn randomly from the HP group has a greater score value than a patient from the non-HP group. p: Probability of the hypothesis that the difference between the two AUCs compared is 0 . _ - electrosyneresis; - - - -: double diffusion.

values (NPV; $81 \%-88 \%$ ) but better in terms of positive predictive values (PPV). In full models, PPV varied $71-75 \%$ with discrete variables and $57-64 \%$ with dichotomous variables. If serological tests were considered as positive when at least one of two scores was positive, Sp did not change but Se was higher. For a HP prevalence of $35 \%$, Se was $76 \%(95 \% \mathrm{CI}$ 0.60-0.92), Sp 82\% (95\% CI 0.74-0.90), PPV 69.5 and NPV 86.4.

After adjustment by disease group, a positive serological result, with discrete variables, was positively associated with exposure $(\mathrm{p}=0.032)$. However, $20 \%$ of subjects with precipitins had no identified exposure and $25 \%$ of subjects without precipitins had exposure. The presence of precipitins tended to be negatively associated with tobacco consumption $(\mathrm{p}=0.08$; table 5).

Eventually, eight false positives (8.8\%) and 11 false negatives $(33.3 \%)$ were obtained. The false positives were as follows. Three idiopathic pulmonary fibrosis (nonexposed), one eosinophilic interstitial pneumonia (exposed farmer), one silicosis (exposed), one left heart failure (nonexposed) and two residual FL (both exposed farmers). The false negatives comprised eight FL, one home HP due to moulds in the walls, one HP due to nonprofessional farming activities (horses) and one HP without identified exposure. The home HP subject was tested for the antigens corresponding to the actual exposure, identified

\section{TABLE 3 Estimated parameters of serological scores with electrosyneresis for full logistic models}

\begin{tabular}{|c|c|c|c|c|c|}
\hline Antigens & Coefficient $\beta$ & $95 \% \mathrm{Cl}$ & p-value & $95 \% \mathrm{Cl}^{\#}$ & $\mathbf{C V}^{\#} \%$ \\
\hline \multicolumn{6}{|l|}{ Discrete variables } \\
\hline Saccharopolyspora rectivirgula & 0.61 & $0.04-1.19$ & 0.04 & $-0.17-1.39$ & 65 \\
\hline Wallemia sebi & 0.33 & $-0.02-0.68$ & 0.06 & $-0.20-0.86$ & 83 \\
\hline Absidia corymbifera & 0.32 & $-0.26-0.90$ & 0.28 & $-0.57-1.21$ & 141 \\
\hline \multicolumn{6}{|l|}{ Dichotomous variables } \\
\hline Intercept & -2.44 & & & & \\
\hline Eurotium amstelodami & 1.04 & $-0.05-2.12$ & 0.06 & $-0.33-2.40$ & 67 \\
\hline Absidia corymbifera & 0.97 & $-0.04-1.97$ & 0.06 & $-0.22-2.15$ & 62 \\
\hline Wallemia sebi & 0.72 & $-0.34-1.78$ & 0.18 & $-0.75-2.20$ & 104 \\
\hline Streptomyces rectivirgula & 0.61 & $-0.38-1.61$ & 0.23 & $-0.63-1.86$ & 104 \\
\hline
\end{tabular}

Cl: confidence interval; $\mathrm{CV}$ : coefficient of variation $\%=\beta / \mathrm{S} \beta \times 100$, with $\mathrm{S}_{\beta}=$ standard error. ${ }^{*}$ : $95 \% \mathrm{Cl}$ and $\mathrm{CV}$ calculated with bootstrap. To interpret this table a patient with, for example, $S$. rectivirgula $=2$ arcs, $W$. sebi $=0$ arcs, $A$. corymbifera $=1$ arc, $E$. amstelodami $=3$ arcs and $S$. mesophilic $=0$ arcs, has the following score with discrete variables: $S_{n}=-2.45+0.61 \times 2+0.33 \times 0+0.32 \times 1+0.16 \times 3+0.14 \times 0=-0.43$, and the following score with dichotomous variables: $S d=-2.44+0.61 \times 1+0.72 \times 0+$ $0.97 \times 1+1.04 \times 1+0.55 \times 0=0.18$. To transform a discrete variable into a dichotomous variable, see table 2 . 


\begin{tabular}{|c|c|c|c|c|c|c|}
\hline \multirow{2}{*}{$\begin{array}{l}\text { TABLE } 4 \\
\text { Variable }\end{array}$} & \multicolumn{6}{|c|}{$\begin{array}{l}\text { Cut-off values (determined with the optimal criterion method }{ }^{\#} \text { ) and performances of serological scores with } \\
\text { electrosyneresis }\end{array}$} \\
\hline & Prevalence \% & Positive serology & Se & Sp & PPV & NPV \\
\hline \multirow[t]{3}{*}{ Discrete } & 20 & $>-0.35$ & 49 & 95 & 71 & 88 \\
\hline & 25 & $>-0.44$ & 55 & 93 & 72 & 86 \\
\hline & $35^{\circ}$ & $>-0.53$ & 61 & 89 & 75 & 81 \\
\hline \multirow{2}{*}{ Dichotomous } & 25 & $>-0.76$ & 60 & 85 & 57 & 86 \\
\hline & $35^{\circ}$ & $>-0.78$ & 66 & 80 & 64 & 81 \\
\hline
\end{tabular}

Se: sensitivity (probability of a positive test outcome in a hypersensitivity pneumonitis (HP) individual); Sp: specificity (probability of a negative test outcome in a non-HP individual); PPV: positive predictive value (probability of having HP when serological score is above the cut-off value); NPV: negative predictive value (probability of having HP when serological score is equal to or below the cut-off value); ND: nondiscriminative. ${ }^{*}: S=((1-P) / P)((C F P-C T N) /(C F N-C T P))$, where $S$ is the slope of the receiveroperating characteristic curve at the optimal operating point, in which "optimal" is in terms of minimising costs (C). "Costs" can be identified here as patient morbidity, where CFP, CFN, CTN and CTP represent the costs of false-positive, false-negative, true-negative and true-positive results, respectively. Here, it was assumed that $\mathrm{CFP}=\mathrm{CFN}$ and $\mathrm{CTP}=\mathrm{CTN}$. Therefore, here $\mathrm{S}=((1-\mathrm{P}) / \mathrm{P})$, where $\mathrm{P}$ denotes the prevalence in the target population. " : prevalence in study population. To use this table for a given patient from, for example, a department of respiratory disease where the prevalence of HP is nearly $35 \%$, with a serological score of -0.43 (>-0.53) with discrete variables and 0.18 (>-0.78) with dichotomous variables, both serological scores indicate a diagnosis in favour of HP. To calculate scores, see table 3 .

by microbiological sampling in the subject's home. This additional investigation showed at least two arcs of precipitation with electrosyneresis for Fusarium verticilloides, Cladosporium sp. and Trichoderma sp. The same investigations were carried out for the nonprofessional farming HP female and Saccharomonospora viridis was identified as the probable responsible antigen (four arcs of precipitation). Six out of the eight false-negative FL results had at least two arcs for hay extracts and a significant number of arcs with electrosyneresis for at least one of the present study's panel of antigens (without reaching a positive score), and the presence of 1-3 arcs of precipitation for at least two of the following antigens that were not in the present study's panel: Aspergillus nidulans, Streptomyces griseoflavus, Thermoactinomyces vulgaris, S. viridis, Rhodotorula rubra, Fusarium solani and Penicillium sp.

\begin{tabular}{|c|c|c|c|c|}
\hline \multirow[t]{2}{*}{ TABLE 5} & \multicolumn{4}{|c|}{$\begin{array}{l}\text { Serological results of patients with } \\
\text { electrosyneresis }{ }^{\#} \text { according to exposure and } \\
\text { tobacco consumption }\end{array}$} \\
\hline & $\begin{array}{l}\text { Positive } \\
\text { serology }\end{array}$ & $\begin{array}{l}\text { Negative } \\
\text { serology }\end{array}$ & p-value & p-value \\
\hline $\begin{array}{l}\text { Patients n } \\
\text { Exposure }\end{array}$ & \multicolumn{3}{|c|}{ Exposure } & \\
\hline Yes & $21(80.8)$ & $22(25.6)$ & $<0.0001$ & 0.032 \\
\hline No & 5 (19.2) & $64(74.4)$ & & \\
\hline \multicolumn{5}{|l|}{ Tobacco } \\
\hline Smoker & $3(11.5)$ & $40(47.0)$ & 0.001 & 0.08 \\
\hline Nonsmoker & $23(88.5)$ & $45(53.0)$ & & \\
\hline
\end{tabular}

Data are presented as $n(\%)$, unless otherwise indicated. *: Full model with discrete variables (see table 3 ), with cut-off associated with a prevalence rate of 35\% (table 4); ${ }^{\uparrow}$ : Cochran-Mantel-Haenszel adjusted to Chi-squared (adjusted by disease group).

\section{DISCUSSION}

In the absence of standardised and validated diagnostic criteria, diagnosis of HP is difficult [1]. Several proposals for diagnostic criteria have been published [19-22] but their diagnostic accuracy has not been tested. Among these criteria, serology has long been considered to be either a minor criterion or a simple marker of exposure [23-26]. However, with typical FL patients, the use of antigens isolated from patients' environments has lead to convincing results [11, 27]. In addition, a recent study by LACASSE et al. [4] identified serum precipitins as a significant predictive factor of $\mathrm{HP}$, regardless of exposure. However, the diversity of antigens and immunological techniques used in this multicentre study did not allow evaluation of the real diagnostic value of precipitins. The HP cohort in the study of LACASSE et al. [4] consisted mainly of BBL disease and subjects were generally tested by ELISA. In the present study, which concerns HP due to moulds, the sera of patients were tested by electrosyneresis and double diffusion using a panel of antigens that included $A$. corymbifera, E. amstelodami, $W$. sebi, three antigens responsible for FL in the region of the present study and rarely considered in other studies. The use of local antigens limits the feasibility of a multicentre study, and it is for this reason that the number of cases in the present study is limited.

The results of the present study showed that it is possible to use a serological score in the diagnosis of HP caused by moulds for patients suffering from an ILD, when an appropriate panel of antigens is used. The results also showed electrosyneresis to be more appropriate than double diffusion for differential diagnosis of HP. However, the sensitivity of the serological test was somewhat disappointing, since about onethird of HP had negative results. However, in eight of the 11 false-negative results for the present study's serological score, the responsibility of antigens not included in the tested panel was either suggested or demonstrated by further antigenic testing. The lack of sensitivity in scores can therefore be explained by the limited panel tested with respect to the great 
diversity of antigens that cause HP. The use of a larger panel of antigens would improve the sensitivity of the methodological approach.

The most discriminative antigens within the tested panel were S. rectivirgula, A. corymbifera, E. amstelodami and W. sebi; the first is recognised as the major aetiological antigen of FL [27] and is responsible for other professional HP due to actinomycetes. The three others are likely to be aetiological factors of FL in Eastern France [11]; however, A. corymbifera and W. sebi have also been shown to be aetiological agents in FL in Scandinavia [28, 29]. Conversely, the present study, as well as the Scandinavian studies [28, 29], have not demonstrated the ability of A. fumigatus to discriminate between HP and non-HP. This antigen has been evoked as an aetiological factor of HP for more than four decades [30] but its actual responsibility in HP is probably rare.

One concern of the present authors is the applicability of the results of the current study. Both the panel of antigens used and the score are not strictly applicable worldwide, mainly because the current ILD cohort includes a high proportion of $\mathrm{HP}$ cases that do not represent the usual aetiologies of HP. However, the approach used, which consisted of developing a serological score through use of a combination of local and international antigens, can be applied worldwide. In addition, it would be useful to test the current panel in other parts of the world. Indeed, this panel probably allows detection of immunological responses induced by a larger number of fungal species and thermophilic actinomycetes, given the known cross-reactivity between different fungal species. In this respect, the present study partially answers the need to develop panels of standardised antigens, highlighted in the recent recommendations of the National Heart, Lung and Blood Institute/Office of Rare Diseases workshop [1].

\section{Conclusion}

Serological tests using a panel of relevant antigens and performed by electrosyneresis help to diagnose mouldinduced hypersensitivity pneumonitis by providing precise estimates of the probability of active disease in a region where hypersensitivity pneumonitis, especially farmers' lung, is frequent.

\section{ACKNOWLEDGEMENTS}

The authors thank Y. Lacasse for his comments on the study protocol, N. Richardson-Peuteuil for proofreading and G. Rival for his assistance in collecting data.

\section{REFERENCES}

1 Fink JN, Ortega HG, Reynolds HY, et al. Needs and opportunities for research in hypersensitivity pneumonitis. Am J Respir Crit Care Med 2005; 171: 792-798.

2 Bourke SJ, Dalphin JC, Boyd G, McSharry C, Baldwin CI, Calvert JE. Hypersensitivity pneumonitis: current concepts. Eur Respir J Suppl 2001; 32: 81s-92s.

3 Selman M. Hypersensitivity pneumonitis. In: Schwarz MI, King Jr TE, eds. Interstitial Lung Disease. Hamilton BC, Decker Inc., 1998; pp. 393-422.
4 Lacasse Y, Selman M, Costabel U, et al. Clinical diagnosis of hypersensitivity pneumonitis. Am J Respir Crit Care Med 2003; 168: 952-958.

5 Respiratory health hazards in agriculture. Am J Respir Crit Care Med 1998; 158: S1-S76.

6 Burrell P, Rylander R. A critical review of the role of precipitins in hypersensitivity pneumonitis. Eur J Respir Dis 1981; 62: 332-343.

7 Mohr LC. Hypersensitivity pneumonitis. Curr Opin Pulm Med 2004; 10: 401-411.

8 Reboux G, Dalphin JC. Hypersensitivity pneumonitis: a technical note on precipitins. Rev Mal Respir 2003; 20: 140-143.

9 Krasnick J, Meuwissin HJ, Nakao MA, Yelandi A, Patterson R. Hypersensitivity pneumontis: problems in diagnosis. J Allergy Clin Immunol 1996; 97: 1027-1030.

10 Aberer W, Woltsche M, Woltsche-Kahr I, Kranke B. IgG antibodies typical for extrinsic allergic alveolitis-an interlaboratory quality assessment. Eur J Med Res 2001; 6: 498-504.

11 Reboux G, Piarroux R, Mauny F, et al. Role of moulds in farmer's lung disease in eastern France. Am J Respir Crit Care Med 2001; 163: 1534-1539.

12 Reboux G, Piarroux R, Bardonnet K, Dalphin JC. Comparison of four serological techniques in immunological diagnosis of farmer's lung disease. Am J Respir Crit Care Med 2001; 163: A747.

13 Edwards JH, Davies BH. Inhalation challenge and skin testing in farmer's lung. J Allergy Clin Immunol 1981; 68: $58-64$.

14 Coleman A, Colby TV. Histologic diagnosis of extrinsic allergic alveolitis. Am J Surg Pathol 1988; 12: 514-518.

15 Pepys J, Jenkins PA, Festenstein GN, Gregory PH, Laceley ME, Skinner FA. Farmer's lung: thermophilic actinomycetes as a source of "farmer's lung hay" antigen. Lancet 1963; 2: 607-611.

16 Kurup VP, Mäntyjärvi RA, Terho EO, Ojanen $\mathrm{TH}$, Kalbfleisch JH. Circulating IgG antibodies against fungal and actinomycete antigens in the sera of farmer's lung patients from different countries. Mycopathologia 1987; 98: 91-99.

17 Greiner M, Pfeiffer D, Smith RD. Principles and practical application of the receiver-operating characteristic analysis for diagnostic tests. Prev Vet Med 2000; 45: 23-41.

18 Carpenter J, Bithell J. Bootstrap confidence intervals: when, which, what? A practical guide for medical statisticians. Stat Med 2000; 19: 1141-1164.

19 Terho EO. Diagnostic criteria for farmer's lung disease. Am J Ind Med 1986; 10: 329.

20 Richerson HB, Bernstein IL, Fink JN, et al. Guidelines for the clinical evaluation of hypersensitivity pneumonitis. $J$ Allergy Clin Immunol 1989; 84: 839-844.

21 Cormier Y, Lacasse Y. Keys to the diagnosis of hypersensitivity pneumonitis: the role of serum precipitins, lung biopsy, and high resolution computed tomography. Clin Pulm Med 1996; 3: 72-77.

22 Schuyler M, Cormier Y. The diagnosis of hypersensitivity pneumonitis. Chest 1997; 111: 534-536.

23 Fink JN, Barboriak JJ, Sosman AJ, Bukosky RJ, Arkins JA. Antibodies against pigeon serum proteins in pigeon breeders. Lab Clin Med 1968; 71: 20-24. 
24 Roberts RC, Wenzel FJ, Emanuel DA. Precipitating antibodies in a midwest dairy farming population towards the antigens associated with farmer's lung disease. J Allergy Clin Immunol 1976; 62: 518-524.

25 McSharry C, Banham SW, Lynch PP, Boyd G. Antibody measurement in extrinsic allergic alveolitis. Eur J Respir Dis 1984; 65: 259-269.

26 Dalphin JC, Toson B, Monnet E, et al. Farmer's lung precipitins in Doubs (a department of France): prevalence and diagnostic value. Allergy 1994; 49: 774-750.

27 Pepys J, Jenkins PA. Precipitin (FLH) test in farmer's lung. Thorax 1965; 20: 21-35.
28 Erkinjuntti-Pekkanen R, Reiman M, Kokkarinen JI Tukiainen HO, Terho EO. IgG antibodies, chronic bronchitis, and pulmonary function values in farmer's lung patients and matched controls. Allergy 1999; 54: 1181-1187.

29 Lappalainen S, Pasanen AL, Reiman M, Kalliokoski P. Serum IgG antibodies against Wallemia sebi and Fusarium species in Finnish farmers. Ann Allergy Asthma Immunol 1998; 81: 585-592.

30 Pepys J, Riddell RW, Citron KM, Cayton YM. Precipitins against extracts of hay and moulds in the serum of patients with farmer's lung, aspergillosis, asthma, and sarcoidosis. Thorax 1962; 17: 366-374. 